\title{
Pulmonary Tumor Thrombotic Microangiopathy Secondary to Extramammary Paget's Disease: An Autopsy Case Report and Literature Review
}

\author{
Akihiro Kato $^{a}$ Hiroshi Kato $^{a}$ Satoshi Komorib $^{b}$ Satsuki Nakano $^{c}$ \\ Takayuki Murase $^{c}$ Motoki Nakamura ${ }^{a}$ Akimichi Morita ${ }^{a}$ \\ ${ }^{a}$ Department of Geriatric and Environmental Dermatology, Nagoya City University Graduate \\ School of Medical Sciences, Nagoya, Japan; ${ }^{b}$ Department of Dermatology, Kyoto Prefectural \\ University of Medicine Graduate School of Medical Science, Kyoto, Japan; 'Department of \\ Pathology and Molecular Diagnostics, Nagoya City University Graduate School of Medical \\ Sciences, Nagoya, Japan
}

\section{Keywords}

Autopsy · Cutaneous malignancy · Extramammary Paget's disease $\cdot$ Pulmonary tumor thrombotic microangiopathy · Skin cancer

\begin{abstract}
Pulmonary tumor thrombotic microangiopathy is a fatal disease secondary to some malignant tumors, such as gastric cancer, esophageal cancer, and pancreatic cancer. A 61-year-old man presented to our clinic with a red plaque with erosion on his scrotum and was diagnosed with extramammary Paget's disease. Seven years after the initial diagnosis, multiple bone metastases were discovered, and he was started on oral administration of tegafur/gimeracil/oteracil. Two years after beginning the oral drug administration, the patient complained of dyspnea. A chest CT scan showed ground-glass opacity. Pulmonary artery blood cytology revealed carcinoma cells. He was diagnosed with pulmonary tumor thrombotic microangiopathy. At 9 days after the diagnosis, he died of circulatory and respiratory failure. The autopsy revealed microscopic metastatic tumor emboli in multiple pulmonary vessels with fibrin thrombus, which confirmed the earlier cytologic diagnosis. Observations in our case were consistent with the typical pattern of this pathology in the lung with multiple metastases. Pulmonary tumor thrombotic microangiopathy with a primary disease of cutaneous malignancy is extremely rare. Here, we report a rare case of pulmonary
\end{abstract}


tumor thrombotic microangiopathy secondary to extramammary Paget's disease with a literature review.

\section{Introduction}

Pulmonary tumor thrombotic microangiopathy (PTTM) is a fatal disease secondary to some malignant tumors that was proposed by von Herbay et al. [1]. In PTTM, tumor embolization induces fibrous intima thickening of the pulmonary arterioles and local coagulation abnormalities, which progress rapidly and are characterized by pulmonary hypertension accompanied by respiratory failure. Because the progression of PTTM is exceptionally rapid, many cases are not diagnosed when the patient is alive, and the diagnosis is confirmed by postmortem pathologic anatomy [2]. Gastric cancer is the most common primary tumor, but some cases have cancer of unknown primary origin, and in other cases, the primary tumor is found after death [3]. It is extremely rare for PTTM to occur with cutaneous malignancy as the primary disease. Here, we report a case of PTTM secondary to extramammary Paget's disease (EMPD), a cutaneous malignancy, and review the literature.

\section{Case Report}

A 61-year-old man, who was referred to our clinic for a red plaque with erosion on his scrotum, was diagnosed with EMPD (Fig. 1a, b). Seven years after his initial diagnosis, multiple bone metastases were discovered, and he was started on oral administration of tegafur/ gimeracil/oteracil. Two years after beginning the drug administration, the patient complained of dyspnea. Echocardiography revealed a high tricuspid regurgitation peak gradient value (28-56 mm Hg), suggesting moderate to severe pulmonary hypertension.

The right heart strain was confirmed by echocardiography. Chest contrast CT did not reveal a pulmonary embolus, but diffuse ground-glass opacity was observed on both sides of the lung field (Fig. 1c). Pulmonary blood flow scintigraphy revealed defects in the peripheral regions of both lungs (Fig. 1d). PTTM was diagnosed on the basis of blood cytology as tumor cells were detected in blood samples collected from the pulmonary artery (Fig. 1f). Low-dose 5 -fluorouracil/cisplatin therapy was administered following the diagnosis of PTTM. On day 8 after the PTTM diagnosis, anemia/thrombocytopenia was observed, and hepatic enzyme levels had progressively increased; therefore, the chemotherapy was discontinued. The next day (day 9), he died of circulatory and respiratory failure. The autopsy revealed diffuse tumor emboli in the arterioles of the lung tissue, and immunostaining results revealed PTTM secondary to EMPD (Fig. 2a-e). In addition, multiple metastases were observed in various sites such as the epicardium, spleen, ureter, adrenal gland, intestinal tract, spine, and systemic lymph nodes.

\section{Discussion}

von Herbay et al. [1] reported 3,300 autopsy cases in which PTTM was present in 21 (3.3\%) of the 630 patients with cancer. The median age of the 21 patients was 61.5 years, and all 21 had distant metastases. Gastric cancer was the most common type of cancer (11 patients,

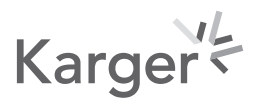




\section{Case Reports in Oncology}

\begin{tabular}{l|l}
\hline Case Rep Oncol 2021;14:1328-1332 \\
\hline DOI: 10.1159/000515262 & $\begin{array}{l}\odot \text { 2021 The Author(s). Published by S. Karger AG, Basel } \\
\text { www.karger.com/cro }\end{array}$ \\
\hline
\end{tabular}

Kato et al.: PTTM Secondary to EMPD

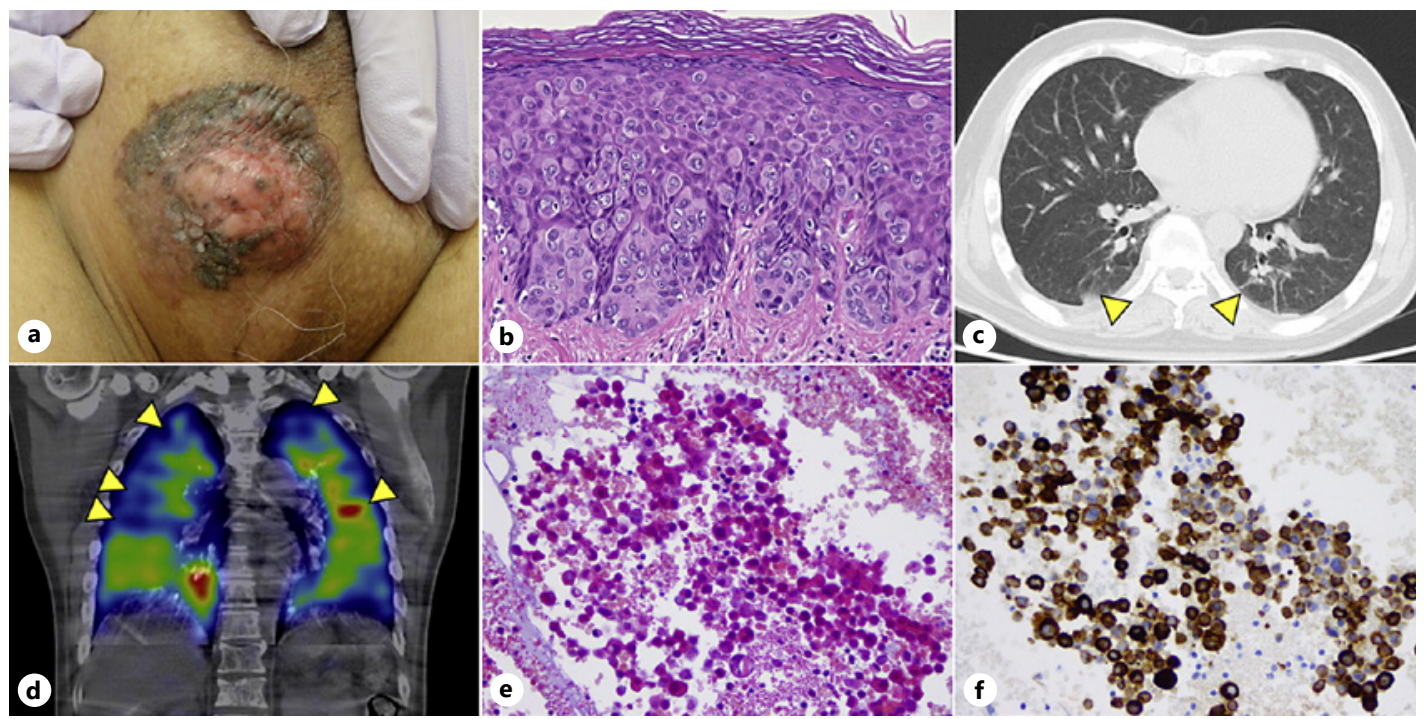

Fig. 1. a Preoperative skin findings: well-defined red to brown plaques are observed. b Pathologic findings of the primary lesion. Paget tumor cells are located in the epidermis. c Computed tomography when dyspnea appeared. No pulmonary embolism is observed, and bilateral diffusely increased leaflet ground-glass-like absorption value is observed.d Pulmonary blood flow scintigraphy. Defects scattered on the peripheral side of the lung field. e Blood cytology (cell block, hematoxylin and eosin staining. $\times 400$ ) collected from the pulmonary artery. Atypical cells with nuclei of different sizes are observed. $\mathbf{f}$ Blood cytology (cell block, cytokeratin 7. ×400). Tumor cells are diffusely positive.

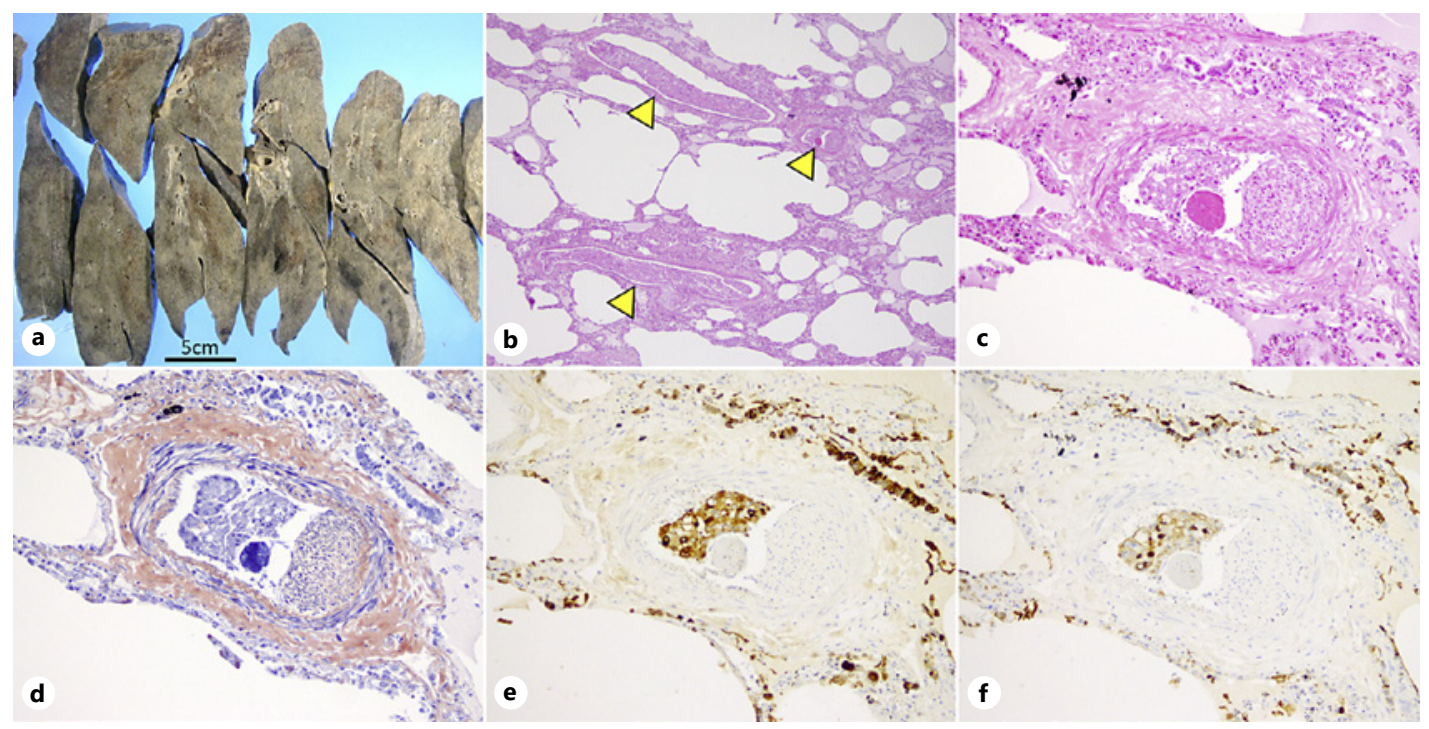

Fig. 2. a Secant image of the lung. Swelling of hilar lymph nodes is observed. b Hematoxylin and eosin staining of lung tissue $(\times 40)$. Tumor embolism in arterioles is observed (arrowhead). c Highly magnified image $(\times 200)$. Eccentric vascular stenosis is observed. d Phosphotungstic acid hematoxylin staining $(\times 200)$. Tumor cells and accompanying fibrin precipitation are observed. e Immunostaining with CAM5.2 $(\times 200)$. Tumor cells are diffusely positive. $\mathbf{f}$ Immunostaining with cytokeratin $7(\times 200)$. Tumor cells are diffusely positive. 
Table 1. Cases of PTTM occurring with cutaneous malignancies

\begin{tabular}{lllll}
\hline & Author & Primary & Diagnosis & Survival \\
\hline 2016 & Banno et al. [7] & EMPD & Blood cytology & 19 days \\
2020 & Oyama et al. [8] & EMPD & Blood cytology, autopsy & 3 days \\
2020 & Our case & EMPD & Blood cytology, autopsy, TBLB & 9 days \\
\hline
\end{tabular}

Both cases emerged secondary to EMPD, and both patients died shortly after diagnosis. Survival indicates survival time from PTTM diagnosis.

EMPD, extramammary Paget's disease; PTTM, pulmonary tumor thrombotic microangiopathy.

52.4\%) [1]. The pathologic features of PTTM are (1) arteriole fibroblastic intima thickening, (2) tumor embolism, and (3) thrombus formation and recommunication [4]. Tumor embolism occurs at the level of the pulmonary arterioles, followed by thrombus formation due to intimal attachment and intima damage by the tumor cells. In addition, fibrocellular proliferation of the intima of blood vessels results in fibrosis, which leads to narrowing and occlusion of the vascular lumen. Pulmonary hypertension and disseminated intravascular coagulation proceed and rapidly progress. Many patients die within a few weeks after the appearance of dyspnea. In PTTM, tumor cells not only cause vascular occlusion but also the local release of factors such as tissue factors that lead to thrombosis due to activation of the coagulation system, inflammatory mediators, and vascular endothelial growth factors. Growth factors are thought to cause fibroproliferative changes in the vascular lumen, progressing to vascular stenosis [5]. Macrophages cluster around blood vessels, and signal transduction between macrophages, tumor cells, and vascular endothelial cells via osteopontin and CD44 is considered to greatly contribute to the onset of PTTM [6]. Only 2 reports of PTTM secondary to cutaneous malignancy, both of which were EMPD, have been published (Table 1) $[7,8]$.

EMPD is a primary malignancy of the skin, and because most extramammary Paget's lesions are detected early, the prognosis is generally favorable. Patients with metastases in which 2 or more lymph node metastases are observed have a worse prognosis [9]. Chemotherapy is used to treat advanced EMPD cases, but to the best of our knowledge, there are currently no data on overall survival [10]. The combination of carcinoembryonic antigen and cytokeratin 19 fragments provides high sensitivity for lymph node metastasis [11]. Carcinoembryonic antigen is mainly detected in cancer tissues such as gastric cancer. On the other hand, cytokeratin 19 fragments are widely used as a tumor marker for epithelial tumors such as lung cancer, breast cancer, and ovarian cancer and is highly specific because it is not produced by nontumor cells. EMPD is a cutaneous malignant tumor, and the expression of both tumor markers was confirmed. On this basis, EMPD might exhibit 2 cytologic properties: squamous epithelium and glandular epithelium. Therefore, EMPD may be present in an environment that makes it more likely than other cutaneous malignancies to cause PTTM, similar to gastric cancer. Although PTTM is a rare complication associated with cutaneous malignancies, it progresses rapidly and has a very poor prognosis. In some cases, PTTM patients are saved by early intervention [12]. Therefore, especially in patients with advanced EMPD, PTTM should be kept in mind in cases of rapidly progressing respiratory failure and pulmonary hypertension, and urgent intervention is required.

\section{Statement of Ethics}

The family of the patient signed a written informed consent form to publish his case.

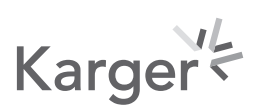




\section{Conflict of Interest Statement}

The authors declare no conflicts of interest associated with this manuscript.

\section{Funding Sources}

The authors declare that there was no funding for this case report.

\section{Author Contributions}

Akihiro Kato, Hiroshi Kato, Satoshi Komori, and Motoki Nakamura were the Dermatologists involved in the case and wrote the case report. Satsuki Nakano and Takayuki Murase were the Pathologists involved in the case and helped in writing and editing the case report. Akimichi Morita supervised the case and helped in writing and editing the case report. All authors read and approved the final manuscript.

\section{Data Availability Statement}

We have his family's permission.

\section{References}

1 von Herbay A, Illes A, Waldherr R, Otto HF. Pulmonary tumor thrombotic microangiopathy with pulmonary hypertension. Cancer. 1990 Aug;66(3):587-92.

2 Uruga H, Fujii T, Kurosaki A, Hanada S, Takaya H, Miyamoto A, et al. Pulmonary tumor thrombotic microangiopathy: a clinical analysis of 30 autopsy cases. Intern Med. 2013;52(12):1317-23.

3 Tanaka M, Takahashi K, Kurihara Y, Yamamoto-Rikitake M, Ogusu S, Hirakawa H, et al. Signet ring cell carcinoma of unknown primary complicated with pulmonary tumor thrombotic microangiopathy and Krukenberg tumor. Case Rep Oncol. 2018 Jun;11(2):412-7.

4 Yamauchi Y, Nakano K, Miyagawa I, Inaba Y, Nawata A, Sato N, et al. An autopsy case of a patient with systemic sclerosis who developed marked pulmonary hypertension because of pulmonary tumor thrombotic microangiopathy caused by gastric carcinoma. Mod Rheumatol Case Rep. 2020 Jan;4(1):56-62.

5 Wakabayashi Y, Iwaya M, Akita M, Takeuchi W, Yamazaki K, Iijima A. Pulmonary tumor thrombotic microangiopathy caused by urothelial carcinoma expressing vascular endothelial growth factor, platelet-derived growth factor, and osteopontin. Intern Med. 2016;55(6):651-6.

6 Higashi A, Dohi Y, Uraoka N, Sentani K, Uga S, Kinoshita H, et al. The potential role of inflammation associated with interaction between osteopontin and CD44 in a case of pulmonary tumor thrombotic microangiopathy caused by breast cancer. Intern Med. 2015;54(22):2877-80.

7 Banno A, Chiba K, Kasai H, Nagami K. Ante-mortem diagnosis of pulmonary tumor thrombotic microangiopathy in a patient with unrecognized extramammary paget's disease. BMJ Case Rep.2016 Aug;bcr2016216666.

8 Oyama Y, Nishida H, Kondo Y, kusaba T, Kadowaki H, Harada T, et al. Pulmonary tumor thrombotic microangiopathy associated with extramammary paget's disease: an autopsy case report. Pathol Int. 2020 Sep; 70(9): $680-5$.

9 Ohara K, Fujisawa Y, Yoshino K, Kiyohara Y, Kadono T, Murata Y, et al. A proposal for a TNM staging system for extramammary paget's disease: retrospective analysis of 301 patients with invasive primary tumors. J Dermatol Sci. 2016 Sep;83(3):234-9.

10 Kato H, Watanabe S, Kariya K, Nakamura M, Morita A. Efficacy of low-dose 5-fluorouracil/cisplatin therapy for invasive extramammary Paget's disease. J Dermatol. 2018 May;45(5):560-3.

11 Kato H, Nakamura M, Watanabe S, Oda T, Morita A. Combined serum carcinoembryonic antigen and cytokeratin 19 fragment levels provide a sensitive biomarker for lymph node metastasis in extramammary Paget's disease. J Dermatol. 2020 Jul;47(7):763-9.

12 Imakura T, Tezuka T, Inayama M, Miyamoto R, Abe A, Otsuka K, et al. A long-term survival case of pulmonary tumor thrombotic microangiopathy due to gastric cancer confirmed by the early diagnosis based on a transbronchial lung biopsy. Intern Med. 2020;59(13):1621-7. 